This is a DRAFT manuscript ("author's electronic pre-print") of:

Rosanna Grassi, Silvana Stefani, Anna Torriero (2010), Centrality in organizational networks, 25 (3), pp 226 - 273

Published Online: Dec 302009 3:20PM

DOI: $10.1002 /$ int.20400

For more information about the Journal, see the publisher's information page at: http://www3.interscience.wiley.com/journal/36062/home 


\title{
Centrality in organizational networks
}

\author{
Rosanna Grassi ${ }^{a}$, Silvana Stefani $^{a}$, Anna Torriero ${ }^{b *}$ \\ ${ }^{a}$ Dipartimento di Metodi Quantitativi per le Scienze Economiche ed Aziendali, \\ Università di Milano-Bicocca, 20126 Milano, Italy \\ ${ }^{b}$ Dipartimento di Discipline Matematiche, Finanza matematica e Econometria. \\ Università Cattolica del Sacro Cuore di Milano, \\ 20123 Milano, Italy
}

\begin{abstract}
In this paper we discuss the role of centrality in organizational networks. We will present some new results related to the different concepts of centrality. A case study of an ICT consulting company concludes.

Keywords: Organizational networks, centrality, graphs
\end{abstract}

\section{Introduction}

Social network analysis can be used to investigate the structure of organizations and to describe formal and informal links among individuals. Collaboration ties, informative flows, personal relationships, advices can be analyzed as organizational networks.

Comparing informal relationships and the formal organizational structure allows us to identify critical points in the firm's performance and to reveal individuals playing a hidden role in the organization, while at the same time confirming important roles of central actors. For a review of literature relevant to organizational networks, see [15], [18], [19], [20], [21]. $[8]$

Among several topics treated in Social Network Analysis we will consider

- roles and positions in a network described by centrality;

- local and global measures suitable to analyze the structure of a network.

In order to clarify network characteristics and the role of each actor in it, we mainly focus on different centrality measures (degree centrality, closeness centrality, eigenvector centrality and betweenness centrality). Centrality is a

\footnotetext{
*Corresponding author. e-mail : rosanna.grassi@unimib.it ; silvana.stefani@unimib.it ; anna.torriero@unicatt.it
} 
structural property, describing the extent to which a given individual is connected to others in a network. It is associated to power, influence in decision making, innovation ([3], [4], [9], [10]).

It is known that individuals with many direct connections to others have more relationships to draw upon in obtaining resources and so are less dependent on any single individual. Centrality also implies control over the resource acquisition of others because central individuals can choose from a greater number of alternative individuals ([20]).

Specific centrality measures can be useful to highlight the position of an individual and his role in the organization. More precisely, we can distinguish four strategic positions in an organization:

1) central individuals: numerous links to others, implying greater opportunities to exchange directly resources and information. Degree centrality is the tool to use here.

2) brokers: individuals playing an intermediary role in the organization through the control of information flows. The number of direct links is not necessarily high, but their betweenness centrality is indeed high.

3) boundary spanners or ambassadors: they communicate especially outside their group with central individuals of other groups in order to establish strategic alliances or to develop new goods: the right measure here is eigenvector centrality.

4) boundary specialists: they own technical skills or specific information, and their position is intentionally on the boundary of the network. These individuals are characterized by a low centrality measure.

Furthermore, in order to identify people sharing the same centrality score, we will consider the notion of center of a network and its properties.

An interesting point is to ascertain when one or more actors can be the most central under other concepts of centrality that we consider. First, we prove a sufficient condition for a most degree central node to be a most eigencentral one. Then we will show that actors with the star property, i.e. actors directly connected to all others in the network, belong to all centers. A case study of an ICT consulting company will show how centrality, related in particular to brokers, can be used to optimally design an organizational network.

In the study of organizational networks, another important issue refers to the concept of structural equivalence ([15], [17], Chapter 9) and core-periphery structure ([7], [4]). The structural equivalence was first introduced in [14] to identify individuals playing similar roles in a network, in other words those who can be swapped without changing the topology of the network. The structural equivalence concept aims at making operational the notion that actors may have identical or nearly identical positions in a network and hence be directly "substitutable" for one another ([13]). A less strict concept, the automorphic equivalence (see [17], Chapter 9) can be observed in many organizational networks and it concerns individuals that are not connected to exactly the same nodes, like those structurally equivalent are, but have the same pattern of ties and similar structural positions, i.e. the network exhibits some "parallel structures" or replicated substructures. In the case study we will discuss automorphic 
equivalence.

Another meaningful property for better understanding the role and behaviour of individuals in organizational networks is the core-periphery structure ( [5]). An intuitive view of the core-periphery structure is the idea of a group or network that cannot be subdivided into exclusive cohesive subgroups or factions, although some actors can be connected better than others. They constitute the core and are related not only to each other, but to all nodes in the network, while actors at the periphery are related only to the core. In the core we find hubs connected to other hubs, as well as hubs in a central position rather than in the network periphery; those hubs are characterized by a high degree centrality. As a result, the network shows a more compact structure and a better speed of information. We observe that this property is generally attributed to scale-free networks. A measure that can help characterize core-periphery structures is the $s$-metric ([1]). In [12] it is proved that trees having the maximum value of $s$-metric have minimum diameter, thereby allowing the fastest information flow. The analysis can be carried out also on groups, units, compartments... Consider for instance an advice network, where actors are related if they share advices in whatever circumstances.

In this paper we will remind the concepts of automorphic and structural equivalence (Section 2); next (Section 3) we will give results related to centrality and quote and prove theorems. We will conclude (Section 4) with the case study of an ICT consulting company. Conclusions are in Section 5.

\section{Preliminaries}

We recall some basic definitions about graphs.

Let $G=(V, E)$ be a graph, where $V$ is the set of vertices with $|V|=n$ and $E$ the set of edges with $|E|=m$; we assume $G$ simple, finite, undirected and connected $^{1}$. A vertex $v \in V$ is a cutvertex if the graph $G \backslash v$ is not connected; $W \subseteq V$ is a cutset if the graph $G \backslash W$ obtained by removing the set of vertices $W$ is not connected.

Denote by $A=\left[a_{i j}\right]$ the adjacency matrix of $G .\left\{\lambda_{1}, \lambda_{2}, \ldots, \lambda_{n}\right\}$ is the set of the eigenvalues of $A, \rho=\max _{i}\left|\lambda_{i}\right|$ its spectral radius and $\mathbf{x}$ the principal eigenvector corresponding to $\rho ; A$ is symmetric, non negative and irreducible and its eigenvalues are real. Two (not) adjacent vertices $v$ and $w$ will be denoted by $v \sim w(v \not w)$.For every $v \in V, N(v)=\{w \in V: w \sim v\}$ is the set of vertices adjacent to $v$. Its cardinality is called the degree of $v$ and denoted by $d(v)$. A complete graph on $n$ vertices denoted by $K_{n}$ is a graph where each pair of vertices are adjacent. The induced subgraph of a set $W \subseteq V$ is the maximal subgraph of $G$ with vertex set $W$, denoted by $\langle W\rangle$. A path from $v_{1}$ to $v_{k}$ is a sequence of distinct adjacent vertices $v_{1}, v_{2}, \ldots, v_{k}$; a shortest path from $v_{1}$ to $v_{k}$ is said to be a geodesic. The distance $d(u, v)$ between two vertices $u$ and $v$ is the length of a shortest path from $u$ to $v$.

\footnotetext{
${ }^{1}$ For these definitions see $[6]$
} 
Two graphs $G=(V, E)$ and $G^{\prime}=\left(V^{\prime}, E^{\prime}\right)$ are isomorphic if there is a bijective function $f: V \rightarrow V^{\prime}$ (called isomorphism) adjacency-preserving, i.e. $u \sim v$ if and only if $f(u) \sim f(v)$. An automorphism on $G$ is an isomorphism $f: V \rightarrow V$.

Definition 2.1 Two vertices $u, v \in V$ are called automorphically equivalent in $G$ if $v=f(u)$ for some automorphism $f$ of $G$.

Definition 2.2 Two vertices $u, v \in V$ are called structurally equivalent in $G$ if, for any vertex $w \in V, w \neq u, v, w$ is a neighbour of $u$ if and only if is a neighbour of $v$.

\section{Centers and centrality measures}

In this section we will introduce centrality measures and related theorems. First we recall the definition of structural index on the set of vertices $V$ ([17], Chapter $3)$ :

Definition 3.1 A structural index is a real-valued function $s_{G}: V \rightarrow \mathbb{R}$ such that if an isomosphism $f: V \rightarrow V^{\prime}$ exists, then $s_{G}(v)=s_{H}(f(v))$.

As a consequence, the definition of centrality measure can be introduced as following:

Definition 3.2 A centrality measure $C$ is a structural index $C: V \rightarrow \mathbb{R}$ that associates to each vertex $v \in V$ a non negative real number $C(v)$ such that, given $v, w \in V, v$ is more central than $w$ if and only if $C(v)>C(w)$. The number $C(v)$ is called the centrality of $v$.

The standard centrality measures can be unified under the more general concept of the previous definition, by the function $C$. For further details about the meaning of these measures, see for example [3].

Degree centrality This is the most intuitive definition of centrality, related to degree. The more ties an actor has, the more power he/she has (i.e. the higher the degree of a vertex, the higher its centrality). Actors who have more ties have greater opportunities because they have choices, since they are less dependent on others. By using the function $C$ :

$$
\forall v \in V \quad C_{\text {deg }}(v)=d(v)
$$

and the normalized degree centrality is $\frac{C_{d e g}(v)}{n-1}$.

Closeness centrality This measure is based on the distance between a vertex and all others (given by the sum of geodesic distances). As far as degree centrality is concerned, power can be exerted by direct bargaining and exchange; but power also comes from acting as a "reference point" and being a center in spreading information. Actors who are reachable by others through a path 
shorter than other actors, are more likely reached because the effort to get an information is less by following a geodesic. By using the function $C$ :

$$
\forall v \in V \quad C_{\text {clos }}(v)=\frac{1}{\sum_{u \in V} d(u, v)}
$$

The normalized closeness centrality is $(n-1) C_{\text {clos }}(v)$.

Betweenness centrality. Betweenness is related with the number of times that a vertex needs another vertex (whose centrality is measured) in order to reach a third vertex via a shortest path. It essentially measures the "intermediary" role in a graph and gives an idea of the volume of traffic/information flowing between any two vertices through the intermediary. This gives the intermediary the capacity to break contacts and to isolate actors or prevent contacts.

$$
\forall v \in V \quad C_{b e t w}(v)=\sum_{u<w} \frac{g_{u w}(v)}{g_{u w}}, \quad u, w \neq v
$$

where $g_{u w}$ is the number of geodesics from $u$ to $w$, and $g_{u w}(v)$ is the number of geodesics between $u$ and $w$ passing through $v$. The normalized betweenness is $\frac{C_{b e t w}(v)}{\left(\begin{array}{c}n-1 \\ 2\end{array}\right)}$.

Eigenvector centrality It is defined as the principal eigenvector of the adjacency matrix. Even if a vertex has a few ties, if those few vertices influence many others (who themselves influence still more others), then the first vertex in that chain is highly influential. In a disease network, if a person has the potential to get a disease from a few neighbours, and those neighbours have high disease risk, the potential risk of getting disease for the first person is still very high.

$$
\forall v \in V \quad C_{e i g}(v)=x_{v},
$$

where $x_{v}$ is the $v-t h$ component of the principal eigenvector $\mathbf{x}$, i.e. $x_{v}=$ $\frac{1}{\rho} \sum_{i=1}^{n} a_{i v} x_{i}, \quad i=1, \ldots, n$. The normalized eigenvector is $\frac{C_{e i g}(v)}{\|\mathbf{x}\|_{2}}$, where $\|\cdot\|_{2}$ is the Euclidean norm.

It can be shown ([3]) that an eigenvector is proportional to the row sums of the matrix $S$ obtained by summing all powers of the adjacency matrix $A$, weighted by corresponding powers of the eigenvalue reciprocal:

$$
S=A+\lambda^{-1} A^{2}+\lambda^{-2} A^{3}+\ldots
$$

The measure counts the number of walks of all lengths, weighted inversely by $\lambda$, which emanate from a vertex. Since the $(i, j)$-entry of $A^{k}$ is the number of walks of length $k$ (not only paths and not only geodesics) from vertex $i$ to vertex $j$, eigenvector centrality measures the possibility to reach a vertex even when following complex walks, but giving less importance to longer walks.

Given any centrality measure $C$, we define: 
Definition 3.3 The center of $G=(V, E)$ is the set:

$$
\varphi(G)=\{v \in V: C(v) \geq C(u), u \in V\} .
$$

The center of $G=(V, E)$ contains all vertices $v$ with maximum value with respect to a centrality measure; obviously, all standard centrality measures can be related to the concept of a center. More precisely, $\varphi_{\text {deg }}, \varphi_{\text {betw }}, \varphi_{\text {eig }}, \varphi_{\text {clos }}$, will be associated to $C_{d e g}, C_{b e t w}, C_{e i g}$ and $C_{\text {clos }}$ respectively. The centers do not necessarily coincide, i.e. nodes who belong to the center for a given centrality measure may not belong to the center for another measure.

For this reason is can be interesting to find necessary and/or sufficient conditions to guarantee that a node or nodes belong to more centers. In [10] the following conditions for nodes belonging to $C_{d e g}$ to belong to $C_{e i g}$ are given:

Theorem 3.1 Let $\left(d_{1}, d_{2}, \ldots, d_{n}\right)$ be the degree sequence in nonincreasing order and let $\delta$ be the second highest degree and $\rho$ the spectral radius of the adjacency matrix $A(G)$. If $\rho>\delta$, then $d_{v}=d_{1}$, where $v$ is a node such that $x_{v}=\max _{i \in V} x_{i}$.

Theorem 3.2 Let $d_{2}$ be the second highest degree and let $\rho>d_{2}$; then $x_{v}=$ $\max _{i \in V} x_{i}$ if and only if $v$ is the node with maximum degree.

Let us define ([16]):

$$
\operatorname{Star}(G)=\{v \in V: d(v)=n-1\} ;
$$

Vertices $c$ such that $v \in \operatorname{Star}(G)$ are called star vertices.

Definition 3.4 A center $\varphi$ of $G$ satisfies the star property if $\varphi(G)=\operatorname{Star}(G)$.

Thus a center $\varphi$ of $G$ satisfies the star property if the central vertices are exactly those having degree $n-1$.

First we recall the following results. We assume $\operatorname{Star}(G) \neq \varnothing$.

Theorem 3.3 (Grassi et al. [10] ) Let $G=(V, E)$ be a simple, connected and undirected graph, and $v_{i} \in V$ any vertex such that $d\left(v_{i}\right)=n-1, i=$ $1, \ldots, k \leq n$. Then, $\forall i=1, \ldots, k, C_{\text {eig }}\left(v_{i}\right)=x_{v_{i}}=\max _{w \in V} x_{w}$. Moreover, $\forall v \neq v_{i}$, $C_{\text {eig }}\left(v_{i}\right)>C_{\text {eig }}(v)$.

Theorem 3.4 Let $v_{i} \in V$ be any vertex such that $v_{i} \in \operatorname{Star}(G), \forall i=1, \ldots, k \leq$ n. Then, $\forall v \notin \operatorname{Star}(G), C_{\text {betw }}\left(v_{i}\right)>C_{\text {betw }}(v)$.

Proof. Let $v_{i} \in \operatorname{Star}(G)$, then $d\left(v_{i}\right)=n-1$, for $i=1, \ldots, k$. First we will prove that, $\forall v \in V, v \neq v_{i}$, the inequality $C_{\text {betw }}\left(v_{i}\right) \geq C_{\text {betw }}(v)$ is true.

$\forall u, w \in V$, if $u \sim w$ then $d(u, w)=1$; if $u \nsim w$ then $d(u, w)=2$, being $u$ and $w$ both adjacent to $v_{i}$; consequently, the number $g_{u w}$ of geodesics from $u$ to $w$ is $r$, where $r(1 \leq r \leq \min (d(u), d(w))$ is the number of vertices adjacent to both $u$ and $w$. 
Let us consider a vertex $v \neq v_{i}, u$ and $w$. If $u \nsim w$ then $^{2}$ :

$$
g_{u w}(v)=\left\{\begin{array}{cc}
1 & \text { if } v \sim u, w \\
0 & \text { otherwise }
\end{array} ;\right.
$$

(obviously, if $u \sim w$, then $g_{u w}(v)=0$ ), and $\frac{g_{u w}(v)}{g_{u w}}=\frac{1}{r}=\frac{g_{u w}\left(v_{i}\right)}{g_{u w}}$ only if $v \sim u, w$

Now, we compute the betweenness of the vertex $v$ :

$$
\begin{aligned}
C_{b e t w}(v) & =\sum_{u<w} \frac{g_{u w}(v)}{g_{u w}}= \\
& =\sum_{u \sim w} \frac{g_{u w}(v)}{g_{u w}}+\sum_{u \nsim w} \frac{g_{u w}(v)}{g_{u w}}=\sum_{u \nsim w} \frac{g_{u w}(v)}{g_{u w}} \geq 0,
\end{aligned}
$$

where the sum is made over all pairs $(u, w)$ with $u<w$ and $u, w \neq v$.

More precisely, if $\sum_{u \nsim w} \frac{g_{u w}(v)}{g_{u w}}=0$, then $C_{b e t w}(v)=0$ and $C_{b e t w}\left(v_{i}\right) \geq$ $C_{b e t w}(v)$. If $\sum_{u \nsim w} \frac{g_{u w}(v)}{g_{u w}}>0$, then all terms $\frac{g_{u w}(v)}{g_{u w}}$ belong to the sum $\sum_{u \nsim w} \frac{g_{u w}\left(v_{i}\right)}{g_{u w}}$, consequently:

$$
C_{b e t w}\left(v_{i}\right)=\sum_{u \nsim w} \frac{g_{u w}\left(v_{i}\right)}{g_{u w}} \geq \sum_{u \nsim w} \frac{g_{u w}(v)}{g_{u w}}=C_{b e t w}(v) .
$$

Now we prove that, $\forall v \in V, v \in \operatorname{Star}(G)$ if and only if $C_{\text {betw }}\left(v_{i}\right)=C_{\text {betw }}(v)$.

If $d(v)=n-1$, then $v \sim v_{j}$ and every other vertex is adjacent to $v$ and $v_{j}$. Consequently:

$$
C_{b e t w}\left(v_{i}\right)=\sum_{u \nsim w} \frac{g_{u w}\left(v_{i}\right)}{g_{u w}}=\sum_{u \nsim w} \frac{g_{u w}(v)}{g_{u w}}=C_{b e t w}(v) .
$$

On the other hand, let $C_{\text {betw }}(v)=C_{\text {betw }}\left(v_{i}\right)$ and suppose, by contradiction, that $d(u)<n-1$; then, there exists at least one vertex (call it $u$ ) not adjacent to $v . \forall w \in V$, if $u \sim w$, then $d(u, w)=1$; if $u \nsim w$ then the vertices $u, w$ do not contribute to the $C_{\text {betw }}(v)^{3}$. As a consequence, $C_{\text {betw }}\left(v_{i}\right)>C_{\text {betw }}(v)$ and this contradicts the assumption.

In conclusion, the vertices $v_{i}$ such that $d\left(v_{i}\right)=n-1, i=1,2, \ldots, k \leq n$ have the same maximum betweenness, i.e. $v_{i} \in \varphi_{\text {betw }}(G), \forall i=1,2, \ldots, k \leq n$ and $\varphi_{\text {betw }}(G)=\left\{v_{1}, v_{2}, \ldots, v_{k}\right\}$.

Theorem 3.5 If Star $(G) \neq \varnothing$ then $\varphi_{\text {deg }}(G), \varphi_{\text {clos }}(G), \varphi_{\text {eig }}(G), \varphi_{\text {betw }}(G)$ satisfy the star property.

\footnotetext{
${ }^{2}$ In fact, let us suppose that $v \nsim u, w$. If a path passing through $v$, from $u$ to $w$ exists, this is certainly not the $(u, w)$ - geodesic, being the geodesic the path of length 2 passing through $v_{i}$.

${ }^{3}$ If a path between $u$ and $w$ exists passing through $v$, this would certainly not be the shortest one, since the $(u, w)$-geodesic must necessarily include $v_{i}$, being $v_{i}$ adjacent to both nodes and $d(u, w)=2$
} 


\section{Proof.}

- $\varphi_{\text {deg }}(G)=\operatorname{Star}(G)$ : being $\operatorname{Star}(G) \neq \varnothing$, then at least a vertex $v$ such that $d(v)=n-1$ exists and $C_{d e g}(v) \geq C_{d e g}(w) \forall w \in V$. The equality $C_{\text {deg }}(v)=C_{\text {deg }}(w)$ holds if and only if $d(w)=d(v)$, and then $\operatorname{Star}(G)=$ $\varphi_{\text {deg }}(G)$.

- $\varphi_{\text {clos }}(G)=\operatorname{Star}(G)$ : see $[16]$.

- $\varphi_{\text {eig }}(G)=\operatorname{Star}(G)$ : see Theorem 3.3.

- $\varphi_{\text {betw }}(G)=\operatorname{Star}(G)$ : see Theorem 3.4.

Theorem 3.6 Let $v_{i} \in \operatorname{Star}(G)(i=1, \ldots, k)$; then $C_{\text {betw }}(v)=0$ for every vertex $v$ such that $d(v) \leq k+1$.

Proof. Let us define $v_{i_{1}}, v_{i_{2}}, \ldots, v_{i_{k}}$ the vertices such that $d\left(v_{i}\right)=n-1$; let $v \notin \operatorname{Star}(G)$, then $k \leq d(v)<n-1$. If $d(v) \leq k+1$, then $v$ is adjacent to the vertices $v_{i_{1}}, v_{i_{2}}, \ldots, v_{i_{k}}$ and (possibly) another vertex. In both cases, $g_{u w}(v)=$ $0, \forall u, w \neq v_{i_{1}}, \ldots, v_{i_{k}}$, since a path of length 2 does not exist passing for any other vertex; the only paths of length 2 passing through $v$ start from $v_{i_{1}}, \ldots, v_{i_{k}}$, but no one of them is a geodesic, since $v_{i_{1}}, \ldots, v_{i_{k}}$ are all adjacent. As a consequence, $C_{\text {betw }}(v)=0$.

Note that all $v_{i} \in \operatorname{Star}(G)(i=1, \ldots, k)$ are not only automorphically equivalent, but also structurally equivalent. Theorem 3.5 implies that the centers contain only vertices belonging to $\operatorname{Star}(G)$.

The former Theorem is not invertible: that is, under the hypothesis that in the graph there are $k$ vertices with degree $n-1$, vertices $u$ such that their betweenness is zero may be not only those with degree $d(u) \leq k+1$.

Finally we quote some recent results related to the eigenvector centrality and the core-periphery structure, which occurs, tipically when high degree vertices are connected with each others. As mentioned in [4], in these classes of networks, eigenvector centrality is not different from degree centrality. More formally, to measure the extent to which a graph $G$ exhibits this "hub structure", [12], has introduced the $s$-metric $S(G)$, first defined in [1] as:

$$
S(G)=\sum_{\left(v_{i}, v_{j}\right) \in E} d\left(v_{i}\right) d\left(v_{j}\right) .
$$

$S(G)$ is maximized in graphs with a hub structure [2]. In [12] we proved new results for trees with maximum $S(G)$ : eigenvector centrality preserves the ordering induced by the degree centrality and they have minimum diameter among trees with a given degree sequence, showing a most "compact" structure.

In analyizing the betweenness centrality of strategic individuals in organizational networks, e.g. lying in a "bridge" position beetween two o more groups it 
can be useful to introduce the concept of a cutvertex. In [11] we proved two theorems. Theorem 3.7 provides a straightforward computation of the betweenness $\beta_{G}(v)$ of a cutvertex:

Theorem 3.7 Let $G$ be a graph, let $v$ be a cutvertex of $G$ and let $A_{1}, A_{2}, \ldots, A_{k}$ be the connected components of $G \backslash v$. If $k=d(v)$ then $\beta_{G}(v)=\sum_{i<j} a_{i} a_{j}$, being $a_{i}=\left|A_{i}\right|$.

Theorem (3.8) characterizes vertices with zero betweenness:

Theorem 3.8 Let $v \in V$. Then $\beta_{G}(v)=0$ if and only if $\langle N(v)\rangle$ is a complete subgraph of $G$.

Other meaningful results concerning upper and lower bounds of betweenness for cutvertices and cutsets have been provided in [11].

\section{A case study}

Centrality can be used to assess the role of individuals or groups in an organizational network. In particular, if in an organization we find out that some individuals, instead of being of support to the team, are of "disturbance" in transmitting information, the general manager may think of moving to another project team or removing them at all. The object of the analysis will be how to shape optimally a project team. We consider the case study of an Italian ITC consulting company, call it ABC. ABC is a major player in technological and informative consulting. A project team must be designed to implement an ERP (Enterprise Resource Planning) system. ERP can be represented as a unique integrated system, divided in modules to support the main corporate functions (accounting, logistics, production, sales). A specific ABC research team, dedicated to implement each module, is typically composed of a team manager (a consultant) and one or more business analysts. However, since the aim is to design an integrated system, the project must be managed at a global level, since decisions taken at a local level can have unforeseeable effects on the efficiency of the other modules. For this reason the general manager is at the head of the whole project. His activity is to coordinate the project team, keeping contact with the team managers and the client itself. Thus, the general manager must belong to a center, to guarantee the necessary level of integration among modules and prevent that a module finds solutions independently of the general policy. The main role of the general manager is to transmit information, acting as a broker. Since a constant and efficient flow of information must be guaranteed, the centrality measure we use is normalized betweenness. We will see that as soon as the complexity of the project increases, betweenness centrality decreases. Thus, ABC developed a team management strategy to guarantee a central role under any condition.

The basic composition of the project team was originally composed of 5 nodes $\left(M=\right.$ Manager, $C_{1}=$ consultant $1, C_{2}=$ consultant $2, A_{1}=$ analyst 


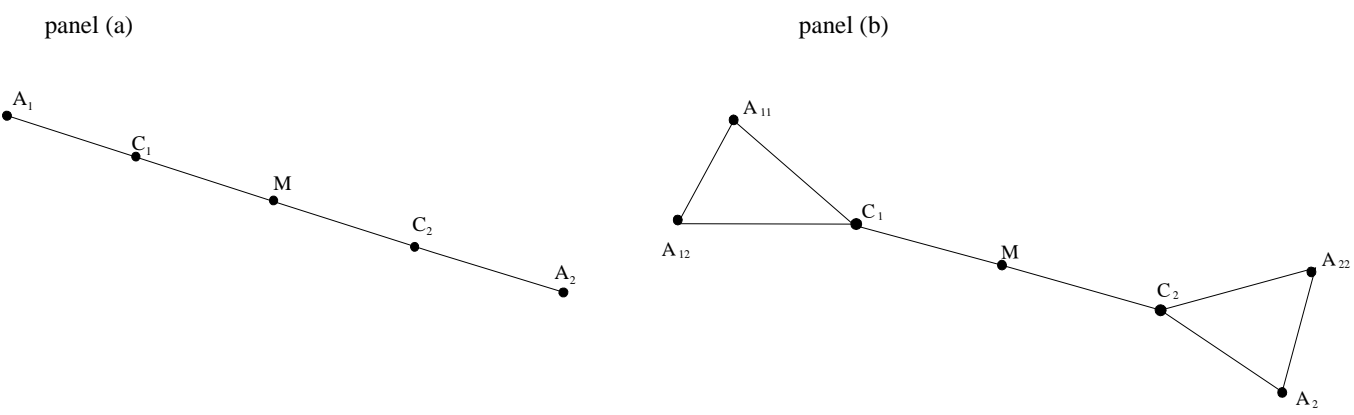

Figure 4.1: Panel (a): Two consultants, two analysts. Panel (b): Two consultants, four analysts.

1, $A_{2}=$ analyst 2) with the corresponding betweenness centralities (see Table 1 and figure 4.1, panel (a)). During the development of the project two new analysts were required, thereby generating a new graph, with the analysts 1 , and 2 on vertices $A_{11}, A_{22}$, and the new analysts 3 and 4 on vertices $A_{12}$ and $A_{21}$ (see Table 2 and figure 4.1, panel (b)).

\begin{tabular}{|l|l|}
\hline Node & Betw \\
\hline$M$ & 0.667 \\
\hline$C_{1}$ & 0.5 \\
\hline$C_{2}$ & 0.5 \\
\hline$A_{1}$ & 0 \\
\hline$A_{2}$ & 0 \\
\hline
\end{tabular}

Table 1 .

\begin{tabular}{|l|l|}
\hline Node & Betw \\
\hline$M$ & 0.6 \\
\hline$C_{1}$ & 0.533 \\
\hline$C_{2}$ & 0.533 \\
\hline$A_{i j, i=1,2, j=1,2}$ & 0 \\
\hline
\end{tabular}

Table 2 .

Observe that $A_{i j, i=1,2, j=1,2}$ have zero betweenness in virtue of Theorem 3.8. Having introduced two new actors, the centrality of the project manager has decreased since $C_{1}$ and $C_{2}$ can communicate directly with two analysts each.

$A_{11}$ and $A_{12}$ are structurally equivalent as $A_{22}$ and $A_{21}$, whereas $C_{1}, A_{11}$, $A_{12}$ are automorphically equivalent respectively to $C_{2}, A_{22}, A_{21}$ and they have the same value of centrality, according to [17], Chapter 3, Definition 3.2.1).

Due to the increasing complexity of the project, two new local teams are introduced, each composed of a consultant and two analysts. Further, a communication flow is established among consultants without necessarily informating the general manager, due to the proximity of functions of the local teams. The manager and the four consultats are the core of a core-periphery structure. The resulting graph is in figure 4.2, panel (a); betweenness measures are in Table 3.

The four consultants belong now to $C_{b e t w}$ while the general manager betweenness has decreased so $M \notin C_{b e t w}$.

A more efficient organizative solution must be found, since the four consultants can bypass him. A possible one is to create two different project teams, 


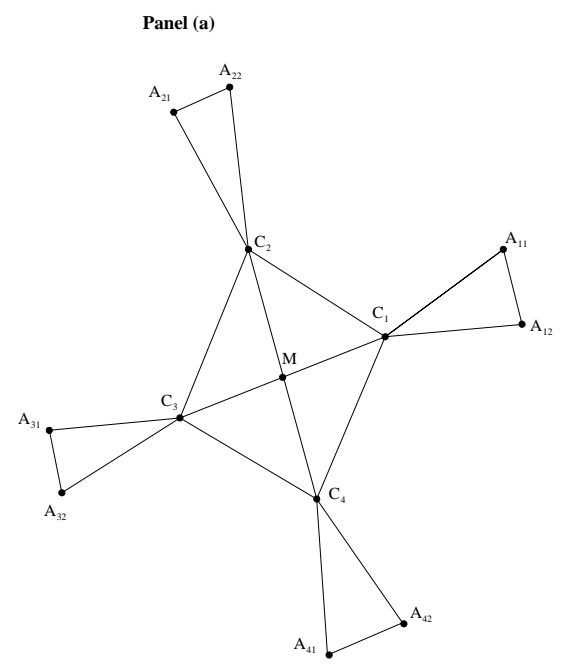

Panel (b)

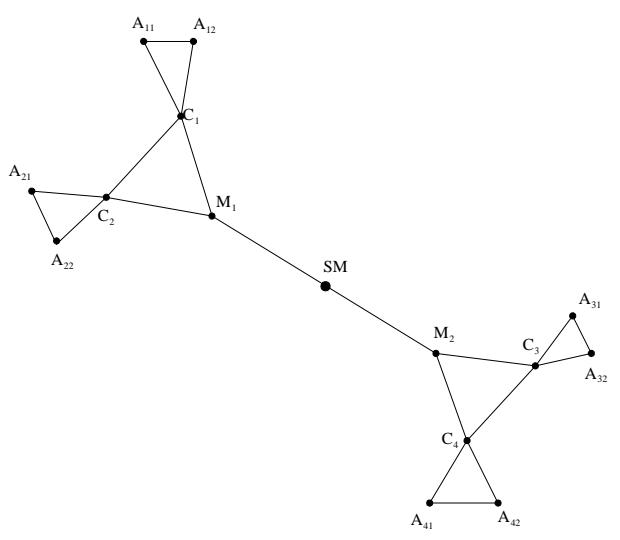

Figure 4.2: Panel (a): Four consultants, eight analysts. Panel (b): Four consultants, eight analysts, two project managers, a senior manager.

under the supervision of a team manager. A senior manager ( $S M$ - the former general manager) will guarantee the necessary flow of information between the two teams. A new graph results with two more nodes; since $S M \in C_{b e t w}, S M$ resumes his centrality role (figure 4.2 and Table 4 ). On the basis of Theorem 3.7, $S M$ betweenness is the product of the cardinalities of the two connected components, obtained by removing $S M$ itself.

\begin{tabular}{|l|l|}
\hline Node & Betw \\
\hline$M$ & 0.091 \\
\hline$C_{1}$ & 0.348 \\
\hline$C_{2}$ & 0.348 \\
\hline$C_{3}$ & 0.348 \\
\hline$C_{4}$ & 0.348 \\
\hline$A_{i j, i=1, \ldots, 4, j=1,2}$ & 0 \\
\hline
\end{tabular}

Table 3.

\begin{tabular}{|l|l|}
\hline Node & Betw \\
\hline$S M$ & 0.538 \\
\hline$M_{1}$ & 0.527 \\
\hline$M_{2}$ & 0.527 \\
\hline$C_{1}$ & 0.264 \\
\hline$C_{2}$ & 0.264 \\
\hline$C_{3}$ & 0.264 \\
\hline$C_{4}$ & 0.264 \\
\hline$A_{i j, i=1, \ldots, 4, j=1,2}$ & 0 \\
\hline
\end{tabular}

Table 4 .

Note how with the increasing complexity of the scenarios, the central role of the general manager has changed and its central role has been progressively declining (see Table 3). However, introducing the new position of a senior manager and splitting responsibilities between two project managers, centrality for the head of the project is restored. Centrality in information can be a serious 
problem in an organization and $\mathrm{ABC}$ solved it by introducing new actors and rearranging tasks and responsibilities.

\section{Conclusions}

In this paper we tried to make a further step in understanding the role of individuals in organizational networks through a graph topological approach based on centrality measures. Our results can be meaningful both on the theoretical side and on the practical point of view, for helping managers in shaping strategic positions in their organizations.

\section{References}

[1] Alderson D L, Li L, Doyle JC, Willinger W. Towards a theory of scale-free graphs: definition, properties, and implications. Internet Math. 2006; 2, n.4: $431-523$.

[2] Alderson DL , Li L. Diversity of graphs with highly variable connectivity. Phys Rev. E 75046102 2007; 1-11.

[3] Bonacich P. Power and centrality: a family of measures. Am. J. Sociol. 1987; 92: 1170-1182.

[4] Bonacich P. Some unique properties of eigenvector centrality. Soc. Networks 2007; 29: 555-564.

[5] Borgatti S, Everett M. Models of core-periphery structures. Soc. Networks 1999; 21: 375-395.

[6] Buckley F, Harary F. Distance in graphs. Redwood City CA: AddisonWesley; 1990.

[7] Cummings J N, Cross R. Structural properties of work groups and their consequences for performance. Soc. Networks 2003; 25 (3): 197-210.

[8] Ferligoj A., P. Doreian, V. Batagelj, Optimizational Approach to Blockmodeling, Journal of Computing and Information Technology, 1996

[9] Freeman LC. Centrality in social networks - conceptual clarification. Soc. Networks 1978; 1: 215-239.

[10] Grassi R, Stefani S, Torriero A. Some new results for eigenvector centrality. J. Math. Sociol. 2007; 33: 237-248.

[11] Grassi R, Scapellato R, Stefani S, Torriero A. Betweenness centrality: extremal values and structural properties. in Naimzada AK, Stefani S, Torriero A Eds. Networks, Topology and Dynamics, Theory and Applications to Economics and Social Systems. Lecture Notes in Economics and Mathematical Systems, 613. Springer Heidelberg, 2008: 161-176. 
[12] Grassi R, Stefani S, Torriero A. Extremal properties and eigenvector centrality of graphs with a given degree sequence, Working Paper n. 165, Department of Quantitative Methods, University of Milano-Bicocca, November (2008).

[13] Hanneman R., M. Riddle, Introduction to social network methods, Cambridge University Press 2005.

[14] Lorrain F, White HC. Structural equivalence of individuals in Social Networks. J. Math. Sociol. 1971; 1: 49-80.

[15] Mizruchi MS, Marquis C. Egocentric, sociocentric, or dyadic? Identifying the appropriate level of analysis in the study of organizational networks, Soc. Networks 2006; 28: 187-208.

[16] Monsuur H, Storcken T, Centers in connected undirected graphs: an axiomatic approach. Op. Res. 2004; 52, n. 1: 54-64.

[17] Network Analysis: methodological foundations. Lecture Notes in Computer Science Vol. 3418 ,Brandes U. Erlebach T. Eds., 2005

[18] Reagans R, Zuckerman EW. Networks, diversity and productivity: the social capital of corporate R\&D Teams. Organ. Sci. 2001; 12: 502-517.

[19] Scott J., Social Network Analysis: A Handbook, London Sage 1991

[20] Sparrowe RT, Liden RC, Wayne SJ, Kraimer ML. Social networks and the performance of individuals and groups. Academy of Management Journal 2001; 44: 316-325.

[21] Wasserman S, Faust K. Social Network Analysis: Methods and applications. Cambridge: Cambridge University Press; 1994. 